\title{
NOTE ON TRANSLATIONS AND TRANSLITERATIONS
}

In general, Greek names have been rendered in their more familiar Latinized form to ease their recognition. Terms such as sungeneia are transliterated unless part of a quotation from an original source. Translations of the Greek quotations, and in one case Latin, are mine unless otherwise indicated. 
THIS PAGE INTENTIONALLY LEFT BLANK 